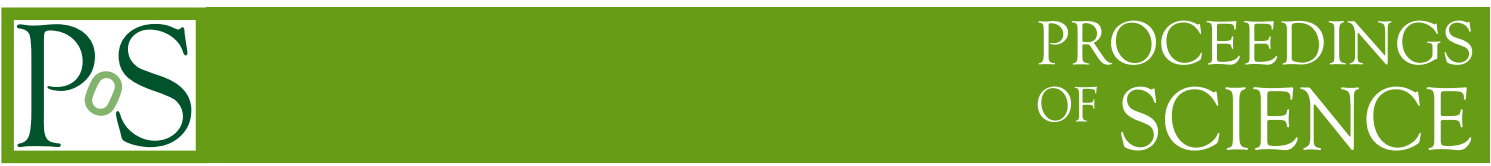

\title{
European Long-baseline Neutrino Oscillation Projects
}

\author{
Marcos Dracos* \\ IPHC, Université de Strasbourg, CNRS/IN2P3, F-67037 Strasbourg, France \\ E-mail: marcos.dracosein2p3.fr
}

\begin{abstract}
After discovering that the last mixing angle $\theta_{13}$ was relatively large, the road is now open to Super Beam projects using conventional accelerator techniques to discover a possible $\mathrm{CP}$ violation in the leptonic sector. These same projects also could resolve the neutrino mass hierarchy problem, if not yet settled by then. For these projects a very intense neutrino beam is required necessitating proton beams with a power higher than an order of magnitude than the present ones. Two european projects are going in this direction, LAGUNA-LBNO and ESS vSB. LAGUNA-LBNO plans to have two stages, one using the present CERN accelerators with improved intensity, to determine the neutrino mass hierarchy using matter effects, and a second one with new CERN installations, to observe CP violation in the leptonic sector. ESSvSB, proposing to use the world's most intense proton linac of the European Spallation Source, operates almost exclusively on the second oscillation maximum, less sensitive to systematic errors. It plans to cover at $5 \sigma$ statistical significance more than $50 \%$ of the $\mathrm{CP}$ violation parameter $\delta_{C P}$. This project, contrarily to LAGUNA-LBNO proposing to use a liquid argon detector, proposes to use a megaton Water Cherenkov neutrino detector installed $1000 \mathrm{~m}$ down in a mine at a distance of about $500 \mathrm{~km}$ from the neutrino source. Both projects have a rich astroparticle physics program and could also study the proton lifetime.
\end{abstract}

16th International Workshop on Neutrino Factories and Future Neutrino Beam Facilities 25 -30 August, 2014

University of Glasgow, United Kingdom

${ }^{*}$ Speaker. 


\section{Introduction}

During the last fifteen years a number of neutrino oscillation projects have been proposed mainly to measure the last unknown mixing angle $\theta_{13}$, determine the neutrino mass hierarchy and observe for the first time a possible CP violation in the leptonic sector. These projects, using the oscillation $v_{\mu} \rightarrow v_{e}$, have mainly been optimised in order to reach as low as possible $\theta_{13}$ values. In 2012, reactor experiments measured for the first time $\theta_{13}[1,2,3]$ and revealed that this value was relatively high $\left(\sim 8^{\circ}\right)$ compared to expectations. Since then, for projects where it was possible, new optimisations have been performed according to the measured $\theta_{13}$ value.

Meanwhile, it has also been shown that for large $\theta_{13}$ values it was better to go to the second oscillation maximum of the oscillation $v_{\mu} \rightarrow v_{e}$, less sensitive to systematic uncertainties [4], for $\mathrm{CP}$ violation discovery. It can also be shown [5] that the neutrino/anti-neutrino asymmetry in the vacuum is approximately equal to $0.30 \sin \delta_{C P}$ at the first oscillation maximum, while for the second oscillation maximum this value becomes $0.75 \sin \delta_{C P}$. This clearly shows that experiments at the second oscillation maximum have significantly higher sensitivity to $\delta_{C P}$ than those placed at the first oscillation maximum.

The drawback of going to the second oscillation maximum compared to the first one comes from the significant decrease of statistics for the same neutrino energy, due to the needed higher distance from the neutrino source to the detector location. On the other hand, decreasing the neutrino energy has another drawback coming from the rapidly decreasing neutrino cross-sections, especially below $1 \mathrm{GeV}$.

LAGUNA-LBNO [6] plans to use a high energy neutrino beam and a long baseline of the order of $2300 \mathrm{~km}$, mainly working on the first oscillation maximum to determine the mass hierarchy using in a first stage a relatively low intensity proton beam. In a second stage, in order to increase statistics and be sensitive to $\mathrm{CP}$ violation, more intensive beam and more voluminous detector is planned.

ESSvSB [7] is almost exclusively devoted on the CP violation discovery operating at the second oscillation maximum. In order to produce a very intensive neutrino beam necessary to go to the second oscillation maximum, ESS $v$ SB proposes to use the very powerful proton beam (5 MW) of the European Spallation Source (ESS) under construction in Lund, Sweden [8]. Due to the relatively short baseline of this project $(\sim 500 \mathrm{~km})$, its sensitivity to the neutrino mass hierarchy is relatively low but not negligible. It is believed that this problem will by solved by the moment when these long term projects will start operation [9].

\section{LAGUNA-LBNO}

The LAGUNA-LBNO project is the continuation of LAGUNA [10], an EU FP7 project studying possible locations in Europe able to host a large underground laboratory devoted to neutrino oscillations and astroparticle physics. LAGUNA-LBNO limits its studies to only three sites giving the highest priority to an underground laboratory located at a distance of $2300 \mathrm{~km}$ from CERN. This underground laboratory located in the Pyhäsalmi mine (Finland), is supposed to mainly host a large liquid argon detector detecting neutrinos produced at CERN. 

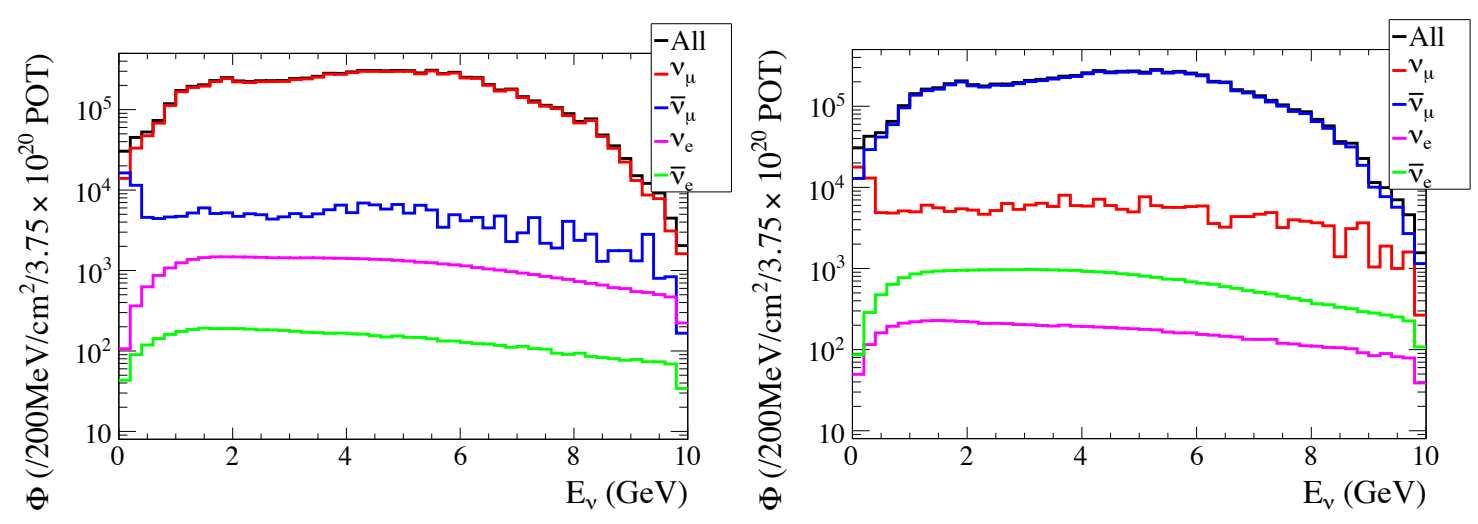

Figure 1: Neutrino (left) and antineutrino (right) fluxes for CERN-to-Pyhäsalmi beam.

In a first phase the present CERN accelerator facilities with improved intensities will be used. A wide band muon neutrino beam can be produced using the SPS proton beam $(400 \mathrm{GeV})$ as done for the CNGS [11]. Fig. 1 [12] presents the neutrino energy distribution for all flavours of the neutrino beam, for the two running modes, "neutrinos" (50\%) and "antineutrinos" (50\%). It is assumed a proton beam power of $750 \mathrm{~kW}$ providing $1.0-1.4 \times 10^{20}$ protons on the target (p.o.t.).

This first phase using a 24 kton liquid argon detector is mainly devoted to the mass hierarchy problem and it is supposed to last about four years $\left(4 \times 10^{20}\right.$ p.o.t. $)$ per year. Fig. 2 presents the statistical significance $T_{0}$ to discover the neutrino mass hierarchy (mean value of $T=\chi_{I H}^{2}-\chi_{I H}^{2}$, comparing normal and inverted hierarchy) versus $\delta_{C P}[6]$. From this figure it is clear that in a short time, more that $5 \sigma$ significance can be obtained for this physics subject for any $\delta_{C P}$ value.
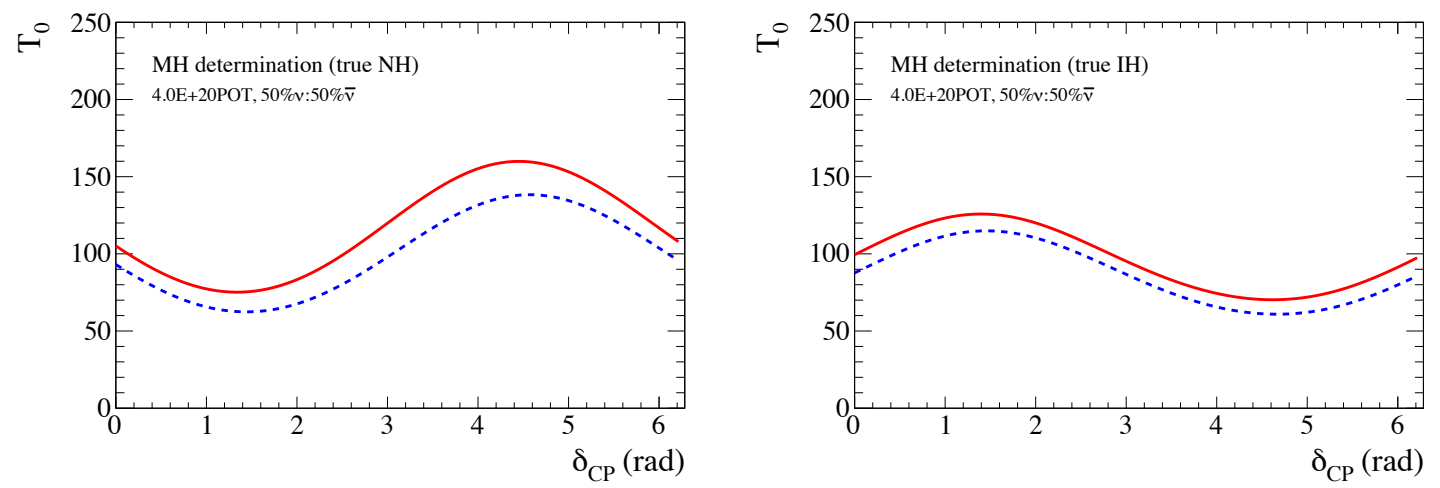

Figure 2: Mean value of the mass hierarchy test statistic $T_{0}$ for nominal and optimised SPS neutrino beams as a function of true $\delta_{C P}$ value.

In a second phase, not using anymore the CERN SPS, but using new powerful CERN accelerators as the low power SPL [13,14] and the High Power PS (HPPS) [15], LAGUNA-LBNO will continue its physics programme mainly devoted to the observation of an eventual CP violation in the leptonic sector. In this phase, a larger detector will be used ( $\sim 70 \mathrm{ktons})$.

The power of protons extracted from the HPPS is expected to be of the order of $2 \mathrm{MW}(3.5 \times$ 

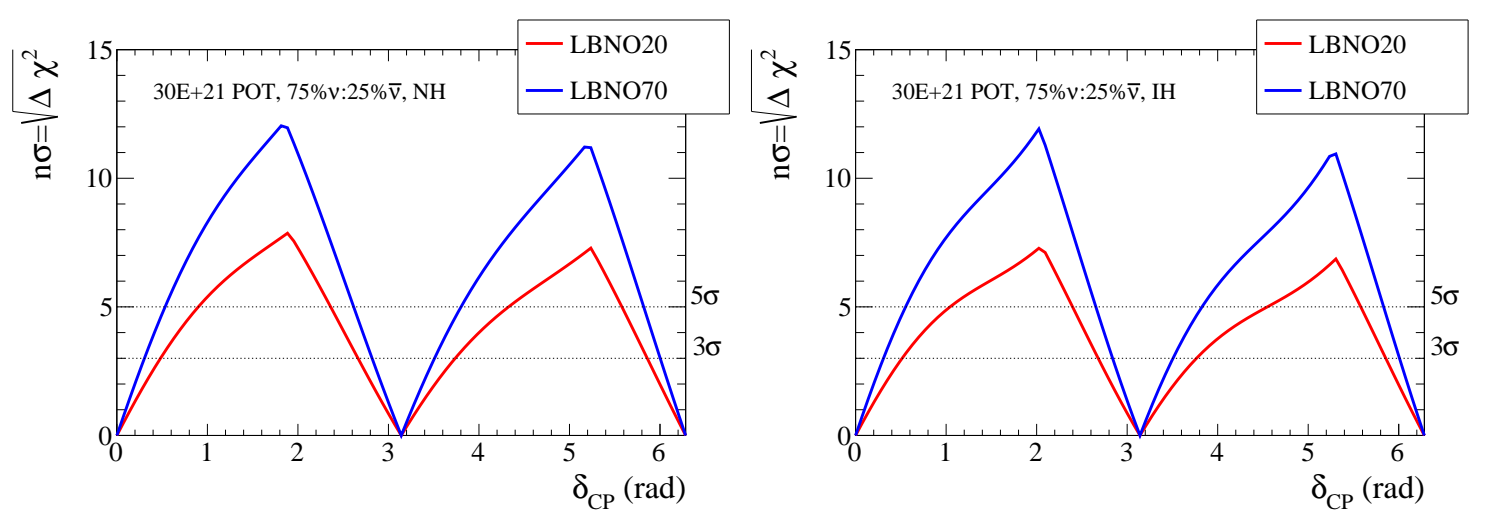

Figure 3: Sensitivity to CPV for the SPL/HPPS beam (normal hierarchy on the left and inverted on the right).

$10^{21}$ p.o.t./year) for $50 \mathrm{GeV}$ proton energy. Fig. 3 shows the discovery potential for $\mathrm{CP}$ violation of this project versus $\delta_{C P}$ for about 10 years of data taking for normal and inverted mass hierarchy. FRom this figure it is clear that a very large liquid argon detector is needed.

This study, done in the framework of the FP7 european projects, is now finished since August 2014. An R\&D program has now started at CERN (WA105) on liquid argon detectors using a two-phase detection technique to prove the feasibility of this large detectors.

\section{3. $\mathbf{E S S} v \mathbf{S B}$}

This second European project is based on the European Spallation Source (ESS) facility under construction in Lund, Sweden. It is exclusively devoted to the $\mathrm{CP}$ violation discovery in the leptonic sector and uses entirely the second oscillation maximum advantages. For this, the very powerful proton source of ESS is necessary.

The ESS is a European facility to provide slow neutrons to research institutes and to the industry. For that, it utilises a very powerful $5 \mathrm{MW}$ linac producing $2 \mathrm{GeV}$ protons running at $14 \mathrm{~Hz}$. The main characteristics of this linac are given in Table 1 . The number of protons on target per year (208 days) is of the order of $2.7 \times 10^{23}$.

Table 1: Main ESS proton linac parameters.

\begin{tabular}{lc}
\hline Parameter & Value \\
\hline Average beam power & $5 \mathrm{MW}$ \\
Proton kinetic energy & $2.0 \mathrm{GeV}$ \\
Average macro-pulse current & $62.5 \mathrm{~mA}$ \\
Macro-pulse length & $2.86 \mathrm{~ms}$ \\
Pulse repetition rate & $14 \mathrm{~Hz}$ \\
Annual operating period & $5000 \mathrm{~h}$ \\
Reliability & $95 \%$ \\
\hline
\end{tabular}


ESS $v$ SB proposes to increase the linac duty cycle in order to double the linac average power (without increasing the instantaneous power) and use half of the produced protons to produce neutrinos. Indeed, the linac duty cycle for neutron production is only $4 \%$. This low duty cycle can be raised to $8 \%$ for simultaneous neutron and neutrino production. To achieve this, the pulse frequency of the linac can be raised from $14 \mathrm{~Hz}$ to $28 \mathrm{~Hz}$, other scenarios are also under study. In this way, it can be sent alternatively, one proton pulse on the neutron target and one on the neutrino one.

Unfortunately, the proton pulse duration of $2.86 \mathrm{~ms}$ is too long for the neutrino production. The necessary current to be sent to the horn in order to well focus the charged pions, coming out of the target, towards the neutrino detector, is of the order of $350 \mathrm{kA}$. Due to this very high current the proton pulses sent to the neutrino facility target with a frequency of $14 \mathrm{~Hz}$ must be as short as possible in order to leave enough time to dissipate the power sent to the horn before the next pulse.

An accumulation ring is necessary, which short circumference could reduce the $2.86 \mathrm{~ms}$ pulses to few $\mu$ s affordable by the horn. To avoid space charge effects during the entrance in the ring, the injection in the linac of $\mathrm{H}^{-}$instead of protons is necessary. In order to fit in the already allocated ESS area, this accumulation ring must have a circumference not longer than $400 \mathrm{~m}$ shortening the proton pulses to about $1.5 \mu \mathrm{s}$, very suited to the horn operation. At the entrance of the accumulator ring the $\mathrm{H}^{-}$ions have to be stripped using a laser-stripping device. Due to the very high beam power, the use of a foil stripping will probably be impossible because the foil would not resist to the proton beam.

On top of the accumulation ring, a target/horn station will also be needed together with a hadron decay tunnel. The decay tunnel length could be of the order of $25 \mathrm{~m}$, long enough to allow charged pions to decay into neutrinos and muons, but also short enough to avoid muon decays (producing electron neutrinos) polluting the muon neutrino beam. Fig. 4 shows a possible layout of the ESS installations and the extra installations necessary to the neutrino beam production.

In order to mitigate the very high power of the proton beam, a system of 4 targets/horns pulsed one after the other is foreseen. This system has been well studied by the FP7 Design Study EURO $v[16,17]$. The EURO $v$ choices have also been adopted for the target and horn cooling. A target of titanium spheres of few mm diameter with cold helium gas cooling is proposed. The design of the horn pulse generator can be found in [18].

The length of the target, the shape of the horn and the length of the decay tunnel have been optimised in order to maximise the discovery probability of CP violation. Fig. 5 presents the neutrino beam composition before oscillation obtained using the ESS $2 \mathrm{GeV}$ proton beam. The mean neutrino energy is of the order of $400 \mathrm{MeV}$. The obtained $v_{\mu}$ beam has an about $0.5 \% v_{e}$ contamination. These electron neutrinos could be used by a near detector to measure the electron neutrino cross-section at the same energies than the electron neutrinos detected by the far detector. These measurements will help to significantly reduce the systematic errors of this project.

The foreseen far detector is a megaton Water Cherenkov similar to MEMPHYS [19, 20]. A Water Cherenkov detector, compared to other detection technics as using a liquid argon or a liquid scintillator detector, is well suited at these relatively low neutrino energies. Its fiducial volume would be of the order of 500 ktons. Several active mines in Sweden are under investigation which could house the far detector. The two most interesting are those of Zinkgruvan and Garpenberg located at $360 \mathrm{~km}$ and $540 \mathrm{~km}$, respectively from Lund. Another interesting mine (Kongsberg) is located in Norway near Oslo at $500 \mathrm{~km}$. To choose the best, one several parameters are taken into 


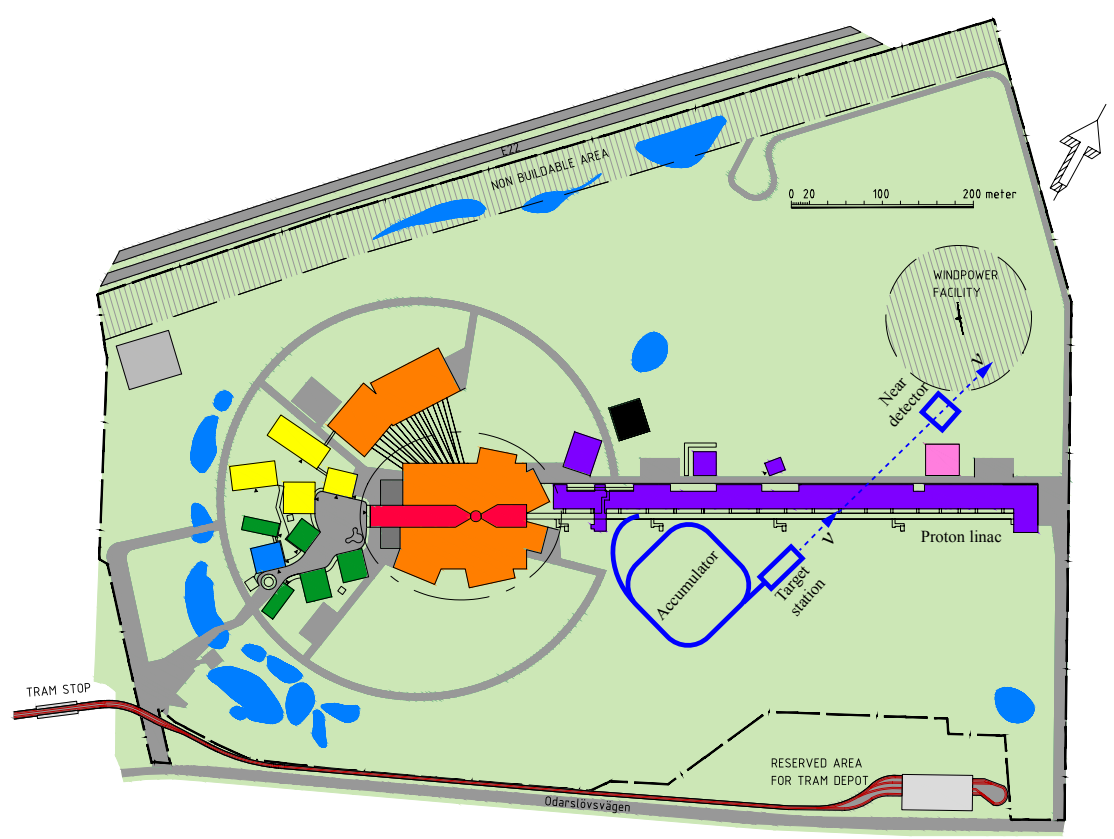

Figure 4: Layout of the ESS installations with a possible neutrino facility implementation.
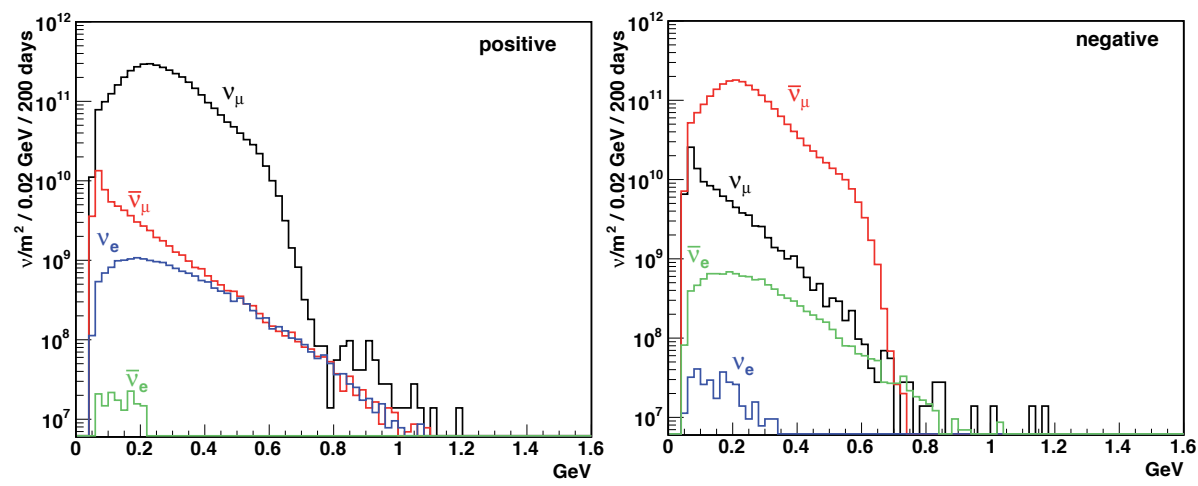

Figure 5: Neutrino energy distribution at a distance of $100 \mathrm{~km}$ on-axis from the target station, for $2.0 \mathrm{GeV}$ protons and positive (left, neutrinos) and negative (right, antineutrinos) horn current polarities, respectively.

account as the situation of the mine itself in order to minimise the civil engineering and increase the physics performance to discover CP violation in the leptonic sector according to the proton beam energy. In this project, resolving the mass hierarchy problem is considered as a secondary physics subject although a $5 \sigma$ discovery significance can be reached for normal and inverted mass hierarchy [7]. It is very likely that this problem will be solved before these next generation long baseline projects. The same far detector can also be used to observe proton decays and to study cosmological neutrinos (from supernova explosions, solar and atmospheric neutrinos etc.).

Fig. 6 shows the neutrino and antineutrino spectra after oscillation (assuming $\delta_{C P}=0$ ) and detected by MEMPHYS detector placed at a distance of $540 \mathrm{~km}$ from Lund. In order to compare 
neutrinos with antineutrinos and to have about the same statistics for both species, it is planned to run 2 years with neutrinos (positive polarity in the horn) and 8 years with antineutrinos (negative polarity).
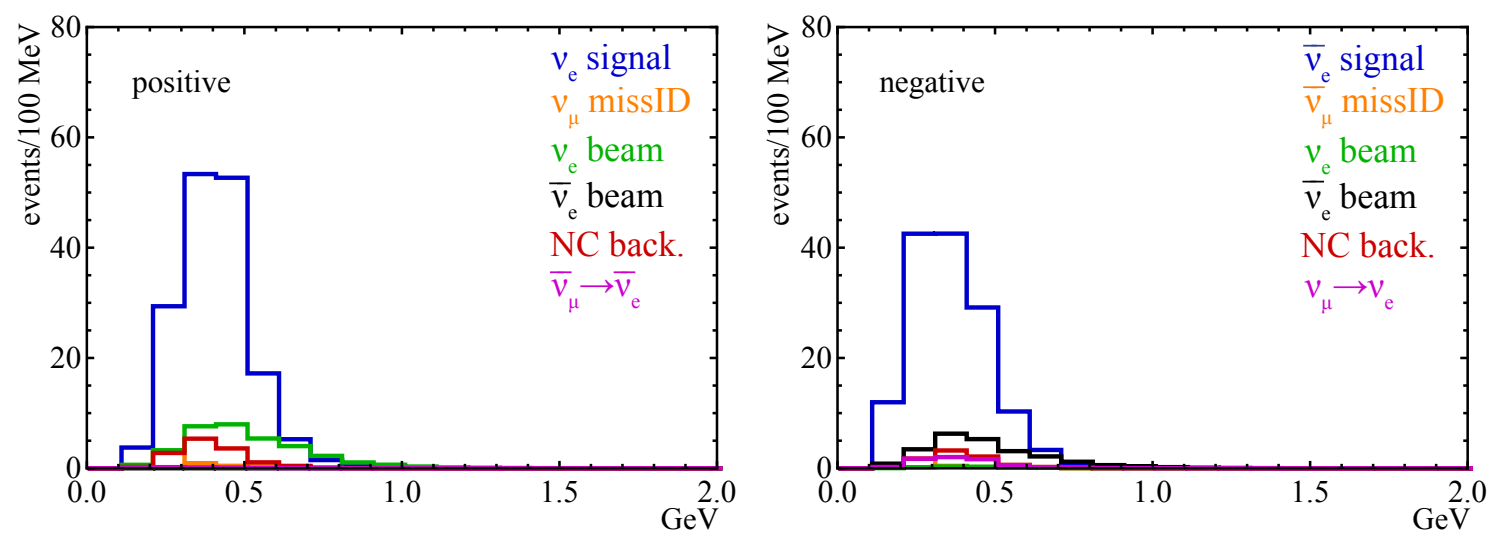

Figure 6: Energy distribution of the detected neutrinos and antineutrinos as reconstructed by MEMPHYS WC detector for two years of neutrino running (left) and eight years of antineutrino running (right) and a baseline of $540 \mathrm{~km}\left(2.0 \mathrm{GeV}\right.$ protons, $\left.\delta_{C P}=0\right)$.

From Fig. 6 it is seen that the background is relatively low for both running modes, neutrinos and antineutrinos. This energy spectrum allows to fully exploit the advantages of the second oscillation maximum, since, as shown by Fig. 7, it completely covers this second maximum.

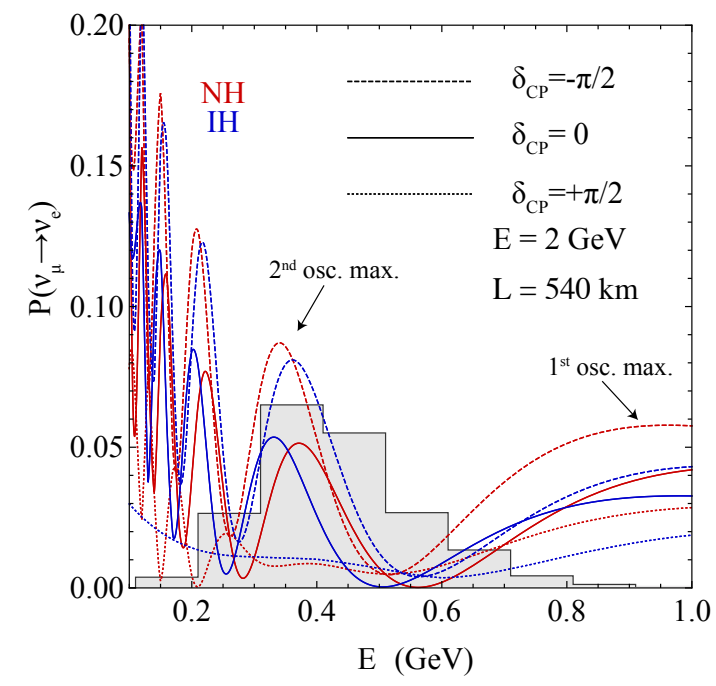

Figure 7: $v_{\mu} \rightarrow v_{e}$ oscillation probability as a function of the neutrino energy. The solid lines are for normal hierarchy $(\mathrm{NH})$ while the dashed ones are for inverted hierarchy $(\mathrm{IH})$. The shaded distribution is the energy distribution of electron neutrinos detected by MEMPHYS far detector.

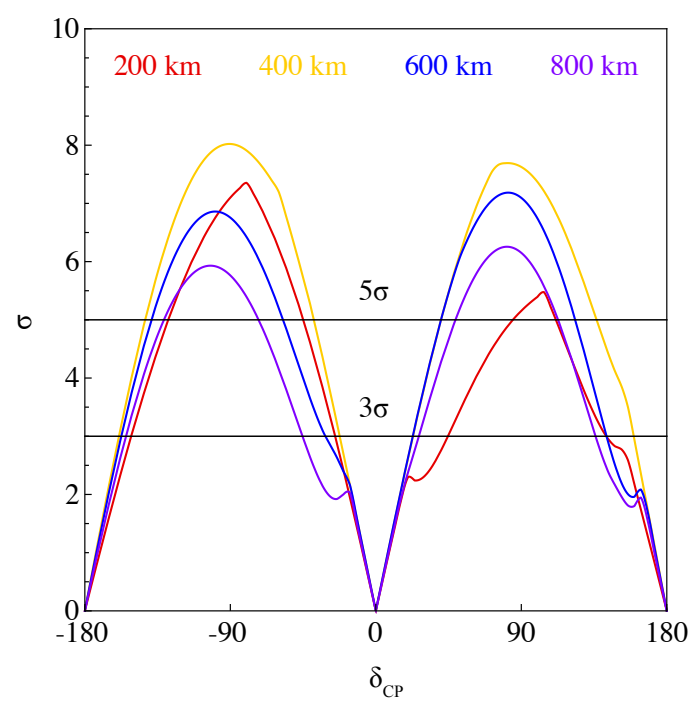

Figure 8: The significance in terms of number of standard deviations $\sigma$ with which $\mathrm{CP}$ violation could be discovered for $\delta_{C P}$ values from $-180^{\circ}$ to $180^{\circ}$ and for different baselines (2.0 GeV protons). 
The discovery probability of $\mathrm{CP}$ violation has been studied as a function of the baseline to find the best distance to place the far detector. Fig. 8 presents the $\mathrm{CP}$ violation discovery significances as a function of $\delta_{C P}$ for several baselines from $200 \mathrm{~km}$ to $800 \mathrm{~km}$. It is seen that this significance can reach values going up to $8 \sigma$ for a baseline of around $400 \mathrm{~km}$ and $\delta_{C P}$ around $-90^{\circ}$ and $90^{\circ}$. These results are obtained assuming normal hierarchy but supposed to be unknown (for inverted hierarchy these results are almost the same). In case the hierarchy is known this performance slightly increases. For the considered baselines matter effects are not expected to play a significant role.

Fig. 9 presents the fraction of the full parameter $\delta_{C P}$ range as a function of the baseline for $3 \sigma$ and $5 \sigma \mathrm{CP}$ violation discovery significance. For $2 \mathrm{GeV}$ protons, the best baseline is around $400 \mathrm{~km}$, close to Zinkgrouvan mine $(360 \mathrm{~km})$. Garpenberg mine (located at $540 \mathrm{~km}$ ) has a better potentiality in case the proton energy goes above $2.5 \mathrm{GeV}$ and can cover up to $60 \%$ of the $\delta_{C P}$ range for a $5 \sigma$ significance. As said above, at this baseline $\operatorname{ESS} v \operatorname{SB}$ fully covers the second oscillation maximum. The first oscillation maximum being around $180 \mathrm{~km}$ has significantly less $\delta_{C P}$ coverage.

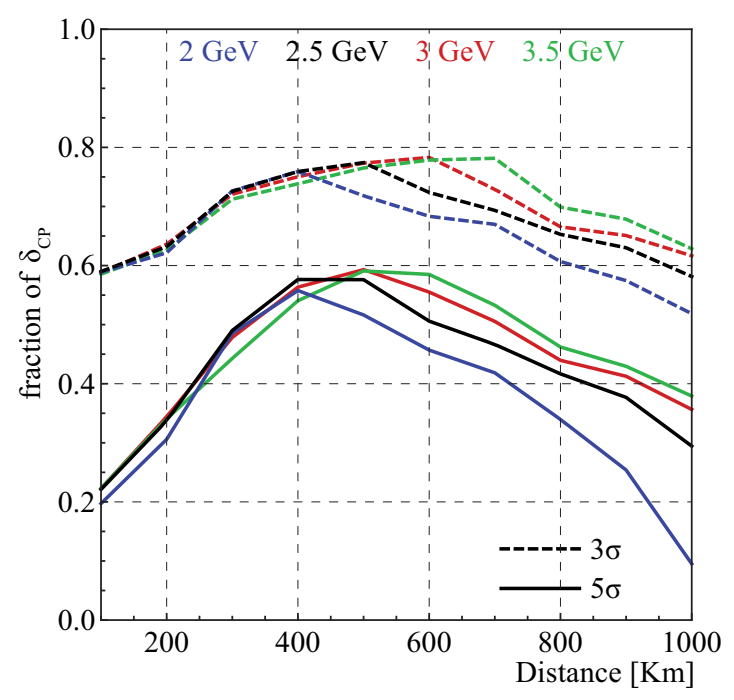

Figure 9: The fraction of the full $\delta_{C P}$ range as function of the baseline. The lower (upper) curves are for $\mathrm{CP}$ violation discovery at $5 \sigma(3 \sigma)$ significance.

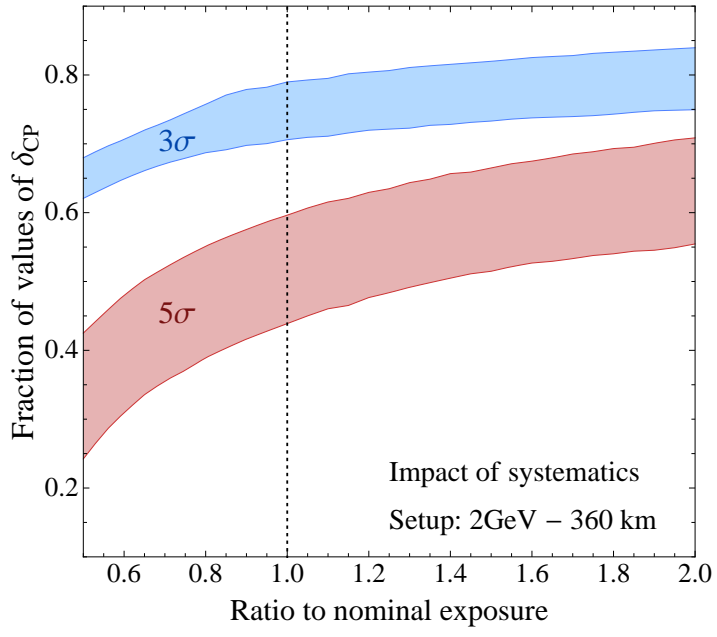

Figure 10: $\delta_{C P}$ coverage as a function of the exposure for different systematic errors, 1 being for 10 years, in case the MEPHYS detector is placed in Zingrouvan mine $(360 \mathrm{~km})$ and for $2 \mathrm{GeV}$ protons.

Fig. 10 [21] shows the $\delta_{C P}$ coverage versus the exposure, 1.0 corresponding to 10 years data taking, for the case of $2 \mathrm{GeV}$ protons and the detector placed in Zingrouvan mine (360 km). The lower limit of this curve is obtained considering the systematic errors mentioned in [22] for Super Beams for the "default" case (mainly assuming 7.5\% systematic error for the signal and 15\% for the background), while the upper limit is obtained assuming the "optimistic" case (mainly assuming $5 \%$ systematic error for the signal and $10 \%$ for the background). It can be seen that for double exposure (20 years running) the $\delta_{C P}$ coverage, at $5 \sigma$ discovery significance, can go up to $72 \%$.

ESSvSB has submitted recently a Design Study project in the framework of the H2020/EU. 


\section{Conclusions}

LAGUNA-LBNO and ESS $v$ SB are the only European long baseline projects proposing to solve the neutrino mass hierarchy problem and observe for the first time a $\mathrm{CP}$ violation in the leptonic sector.

The EU/FP7 LAGUNA-LBNO design study proposes in a first phase to use the existing CERN accelerators with improved performance and a 20 kton liquid argon detector placed in a distance of $2300 \mathrm{~km}$ in a mine in Finland. This first phase is mainly devoted to mass hierarchy while a second phase based on new CERN accelerators, LP-SPL and HPPS, using a larger detector, will be devoted to the CP violation discovery. This design study has ended in August 2014 giving place to an $R \& D$ project on liquid argon detectors.

ESS $v$ SB plans to use ESS installations under construction in Lund and mainly its $5 \mathrm{MW}$ proton linac to produce a very intense neutrino beam in order to discover CP violation. This project fully profites of the developments done in previous European Design studies as EUROV and LAGUNA. This project, due to the neutrino energy and the baseline of about $500 \mathrm{~km}$, will operate exclusively on the second oscillation maximum having enhanced capabilities to discover $\mathrm{CP}$ violation compared to the first oscillation maximum. A megaton Water Cherenkov far detector is considered, placed in one of the already existing mines of the region. For 10 years data taking, $\mathrm{ESS} v \mathrm{SB}$ expects to reach up to $60 \% \delta_{C P}$ coverage at $5 \sigma \mathrm{CP}$ violation discovery significance.

\section{Aknowledgements}

The author would like to thank all ESS vSB colleagues and particularly Pilar Coloma, Enrique Fernandez-Martinez, Tord Ekelof and Nikolaos Vassilopoulos for their help, plots and valuable discussions.

\section{References}

[1] F. P. An et al. [DAYA-BAY Collaboration], "Observation of electron-antineutrino disappearance at Daya Bay,” Phys. Rev. Lett. 108, 171803 (2012) [arXiv:1203.1669 [hep-ex]].

[2] J. K. Ahn et al. [RENO Collaboration], "Observation of Reactor Electron Antineutrino Disappearance in the RENO Experiment,” Phys. Rev. Lett. 108, 191802 (2012) [arXiv:1204.0626 [hep-ex]].

[3] Y. Abe et al. [DOUBLE-CHOOZ Collaboration], "Indication for the disappearance of reactor electron antineutrinos in the Double Chooz experiment,” Phys. Rev. Lett. 108, 131801 (2012) [arXiv:1112.6353 [hep-ex]].

[4] P. Coloma and E. Fernandez-Martinez, "Optimization of neutrino oscillation facilities for large $\theta_{13}$," JHEP 1204, 089 (2012) [arXiv:1110.4583 [hep-ph]].

[5] S. J. Parke, "Neutrinos: Theory and Phenomenology," Phys. Scripta T 158, 014013 (2013) [arXiv:1310.5992 [hep-ph]].

[6] S. K. Agarwalla et al. [LAGUNA-LBNO Collaboration], "Optimised sensitivity to leptonic CP violation from spectral information: the LBNO case at $2300 \mathrm{~km}$ baseline," arXiv:1412.0593 [hep-ph]. 
[7] E. Baussan et al. [ESSnuSB Collaboration], "A Very Intense Neutrino Super Beam Experiment for Leptonic CP Violation Discovery based on the European Spallation Source Linac: A Snowmass 2013 White Paper," Nuclear Physics B, Volume 885, August 2014, Pages 127-149, arXiv:1309.7022 [hep-ex].

[8] The European Spallation Source, http://europeanspallationsource.se/, ESS Technical Design Report, Release 1.0, Nov. 2012.

European Spallation Source Project http://ess-scandinavia.eu

[9] M. Blennow, P. Coloma, P. Huber and T. Schwetz, "Quantifying the sensitivity of oscillation experiments to the neutrino mass ordering,” JHEP 1403 (2014) 028 [arXiv:1311.1822 [hep-ph]].

[10] http://http://laguna.ethz.ch:8080/Plone

[11] Ed. K. Elsener, "The CERN Neutrino beam to Gran Sasso (Conceptual Technical Design)", CERN 98-02, INFN/AE-98/05;

R. Bailey et al., "The CERN Neutrino beam to Gran Sasso (NGS) (Addendum to CERN 98-02, INFN/AE-98/05)”, CERN-SL/99-034(DI), INFN/AE-99/05.

[12] S. K. Agarwalla et al. [LAGUNA-LBNO Collaboration], "The LBNO long-baseline oscillation sensitivities with two conventional neutrino beams at different baselines," arXiv:1412.0804 [hep-ph].

[13] "Conceptual design of the SPL II, A high-power superconducting H- linac at CERN", F. Gerigk et al., CERN-2006-006, 2006.

[14] O. Brunner, S. Calatroni, E. Ciapala, M. Eshraqi, R. Garoby, F. Gerigk, A. Lombardi and R. Losito et al., "Assessment of the basic parameters of the CERN SPL," Phys. Rev. ST Accel. Beams 12 (2009) 070402.

[15] Y. Papaphilippou et al., "Design options of a high-power proton synchrotron for LAGUNA-LBNO", in the proceedings of the 4th International Particle Accelerator Conference (IPAC13), May 12Đ17, Shangai, China (2013).

[16] T. R. Edgecock, O. Caretta, T. Davenne, C. Densham, M. Fitton, D. Kelliher, P. Loveridge and S. Machida et al., 'The EUROnu Project,’ Phys. Rev. ST Accel. Beams 16 (2013) 021002 [arXiv:1305.4067 [physics.acc-ph]].

[17] E. Baussan et al. [EUROnu Super Beam Collaboration], "The SPL-based Neutrino Super Beam," arXiv:1212.0732 [physics.acc-ph].

[18] E. Baussan, E. Bouquerel, M. Dracos, G. Gaudiot, F. Osswald, P. Poussot, N. Vassilopoulos and J. Wurtz, "Study of the pulse power supply unit for the four-horn system of the CERN to Fréjus neutrino super beam,” JINST 8 (2013) T07006 [arXiv:1304.7111 [physics.acc-ph]].

[19] A. de Bellefon, J. Bouchez, J. Busto, J. -E. Campagne, C. Cavata, J. Dolbeau, J. Dumarchez and P. Gorodetzky et al., "MEMPHYS: A Large scale water Cerenkov detector at Frejus," hep-ex/0607026.

[20] L. Agostino et al. [MEMPHYS Collaboration], "Study of the performance of a large scale water-Cherenkov detector (MEMPHYS),” JCAP 1301, 024 (2013) [arXiv:1206.6665 [hep-ex]].

[21] P. Coloma, private communication.

[22] P. Coloma, P. Huber, J. Kopp and W. Winter, "Systematic uncertainties in long-baseline neutrino oscillations for large $\theta_{13}$ ”, Phys. Rev. D 87 (2013) 3, 033004 [arXiv:1209.5973 [hep-ph]]. 